\author{
By opening up its database of potential malaria drugs, GlaxoSmithKline has blazed a path that \\ other pharmaceutical companies should follow.
}

t t was heartening to learn last week that British pharmaceutical company GlaxoSmithKline (GSK) is to make publicly available a database containing the structures and pharmacological data for 13,500 possible drugs against malaria. The database, obtained by screening two million compounds in GSK's library, promises to be a windfall for malaria drug development.

More broadly, the move highlights ongoing efforts by GSK and many other drug companies to re-engage with the 'neglected' diseases that predominantly affect poor countries, reversing decades of indifference. Some firms are already making their libraries available to public-private partnerships such as the Medicines for Malaria Venture (MMV) and the Global Alliance for TB Drug Development. This month's announcement, however, marks the first time that any drug-maker has made public domain a substantial database of possible drug leads.

The move advances the pharmaceutical industry's slow but steady shift towards more open sharing of data. At least for early-stage, precompetitive research, drug companies are finding it useful to lower the firewalls around their intellectual property and pool their resources. Making data public brings fresh eyes and minds to the problem, and has the potential to accelerate the discovery process.

GSK is emerging as a leader in such open-source approaches. Last year, for example, it established a patent pool of drugs and manufacturing processes for neglected diseases (see Nature 457, 1064-1065; 2009) - although in that case, researchers and generic drug-makers could only use the pool for free when working on new treatments for the world's 50 least-developed countries. The exclusion of middleincome countries such as India reflects the reality that GSK and its fellow drug-makers see at least some of the world's poorer nations as potential markets (see Nature doi:10.1038/news.2010.20;2010) altruism has its limits. Yet GSK's actions represent undeniable progress towards a social good - and a welcome about-face for a company that once joined dozens of others in legal action to stop South Africa's government making cheap anti-HIV drugs.

Andrew Witty, GSK's chief executive, deserves credit for showing leadership in this instance. His fellow drug-company chief executives should follow his example. Neglected diseases are a low-risk area for drug companies to experiment with open data, and they should embrace it. But firms need not stop there: such efforts could lead them to rethink their attitudes towards research on diseases of the rich world.

Academic organizations have their own part to play in this movement, by helping to provide the infrastructure for storing and archiving the public data. Good examples of such systems include PubChem, a database of some 27 million compounds that is run by the US National Institutes of Health, and ChEMBLdb, a public drug-discovery database of more than half a million compounds, their targets, and genomic and chemi-

"Neglected diseases are a low-risk area for drug firms to experiment with open data." cal data. ChEMBLdb opened this month in Hinxton, UK, with $£ 4.7$ million (US\$7.6 million) in funding from the Wellcome Trust, and is likely to be an eventual home for GSK's malaria-drug database.

Meanwhile, universities and other academic institutions should do more to support and reward the sort of translational research required to develop drug leads such as those offered by GSK — even though that work usually does not result in high-profile, breakthrough research papers (see go.nature.com/PX8C4m). In addition, such translational activities provide a means for universities to contribute to public-private partnerships such as the MMV, the Drugs for Neglected Diseases Initiative and the Institute for OneWorld Health.

Universities also have another part to play. Their often aggressive intellectual-property policies can stymie research and development in neglected diseases - they should ensure that their licensing deals with companies make exceptions for royalty-free use of technologies for good causes. That change, too, is beginning to happen although, when it comes to hogging intellectual property, academics and their institutions are often among the worst offenders. University heads would do well to emulate Mr Witty.

\section{Valid concerns}

\section{The reporting of candidate biomarkers for disease must be rigorous to drive translational research.}

\footnotetext{
A s the proportion of older people in the global population increases, so too does the burden of chronic conditions such as cardiovascular disease, cancer, Alzheimer's and osteoarthritis. Biological markers that identify disease risk, flag occurrence before symptoms show, determine prognosis or guide treatment choices for individual patients would dramatically reduce this burden.
}

Biomarkers are not a new concept. It is well known that cholesterol levels indicate the risk of cardiovascular disease, that elevated levels of liver enzymes in the blood signal liver damage, and that a particular genetic variation predicts Huntington's disease.

But the past decade has seen a revolution in '-omics' technologies. High-throughput approaches (genome-wide association studies, transcriptomics, proteomics and metabolomics) coupled with bioinformatics are accelerating the pace of biomarker research. It is hoped that these studies will not only reveal new biological 'signatures' of disease to aid diagnosis and the implementation of personalized medicine, but will also shed light on the underlying mechanisms that trigger or perpetuate the condition. 